\title{
Ensino de Emergências na Graduação com Participação Ativa do Estudante
}

\author{
Undergraduate Training on Emergencies \\ with Active Student Participation
}

\author{
Cláudia Regina Fernandes ${ }^{I}$ \\ Sandra Nívea dos Reis Saraiva Falcão \\ Josenília Maria Alves Gomes ${ }^{l}$ \\ Fernando Barros Colares ${ }^{I}$ \\ Maria Márcia Morais Souto Maior ${ }^{I}$ \\ Rafaela Vieira Correa \\ Olívia Andre Alencar Costa Bessal
}

\section{PALAVRAS-CHAVE}

- Métodos;

- Ensino;

- Aprendizagem;

- Competência Clínica;

- Serviços Médicos de Emergência;

- Educação Médica.

\section{KEYWORDS}

- Methods;

- Teaching;

- Learning;

- Clinical Competence;

- Emergency Medical Services;

- Medical Education.

Recebido em: 04/10/2011

Reencaminhado em: 11/09/2012

Reencaminhado em: 01/05/2013

Aprovado em: 10/06/2014

\section{RESUMO}

O ensino médico sofreu intensas modificações nos últimos anos. A rápida evolução do conhecimento requer metodologias que estimulem a metacognição, substituindo o excesso de conteúdo pelo"aprender a aprender", formando profissionais construtores ativos do seu conhecimento e capacitados para refletir sobre a própria prática. A importância do conteúdo de emergências é inquestionável, especialmente na graduação, pois se trata de uma área de grande carência de profissionais que vem sendo cada vez mais ocupada pelo médico recém-graduado, quase sempre despreparado para se conduzir diante da gravidade dos casos. Simular a realidade integra o saber ao fazer, aliados ao desenvolvimento de habilidades. $O$ ensino das emergências em cenários reais é insubstituível, contudo limitado por questões de segurança do paciente e pela impossibilidade de repetição de procedimentos.A simulação em laboratório de habilidades propicia contato do aluno com diversas situações em ambiente controlado, permite repetição $e$ favorece o aprendizado significativo mediante discussão dos temas e reflexão sobre a própria prática.Este artigo descreve a inserção longitudinal do conteúdo de emergências médicas no currículo de um curso de Medicina, utilizando técnicas de simulação e reflexão acerca da prática como metodologia de aprendizado, além da vivência em ambientes de prática real.

\begin{abstract}
Medical education has undergone substantial changes in recent years. The rapid evolution of knowledge demands new teaching models involving active learning, metacognition and reflexive practice. Training in emergency care is of utmost importance in an undergraduate medical course, since the lack of professionals in this area means that emergency care is often staffed by new graduates, almost always unprepared to deal with the severity of the cases. Training technical medical skills within this scenario is necessary to provide the medical student with a realistic vision of the work, however it is limited due to patient safety and the inability to repeat procedures. Teaching emergency care through high fidelity simulators is an excellent solution that allows procedures to be repeated as many times as necessary and favours meaningful learning through discussion of topics and reflections on the practices. This study describes how emergency care training has been included throughout the curriculum for undergraduate medical training in Brazil, using simulation and reflection on the practice as learning tools, as well as real life experience in emergency care settings.
\end{abstract}




\section{INTRODUÇÃO}

Atualmente, ocorre no Estado do Ceará, reflexo do que acontece no Brasil, um caos, um processo de desordem e desorganização nos setores de emergências médicas, resultando na divulgação pela imprensa de números impactantes de morte e invalidez, provocando a criaçãode propostas de efetivação de políticas públicas de saúde voltadas para o atendimento de emergência ${ }^{1}$.

De acordo com o filósofo francês Edgar Morin (1921- ),

[...] a complexidade da relação ordem/desordem/organização surge quando se verifica empiricamente que fenômenos desordenados são necessários, em certas condições, em certos casos, para a produção de fenômenos organizados, que contribuem para o aumento da ordem² (p. 38).

Neste caso, produzir um fenômeno organizado para solucionar a desorganização caótica vigente no cenário das emergências implica necessariamente o desenvolvimento e formação dos recursos humanos que atuam ou atuarão nestes setores, que figuram como área crítica de desempenho dos médicos. Muitos motivos podem ser enumerados como causas desse cenário, entretanto o pequeno número de profissionais adequadamente capacitados merece destaque, principalmente porque parte desta inadequação se deve ao pouco tempo dedicado a este tópico nos currículos de graduação na maioria dos cursos da saúde.

Nas emergências de hospitais de porte primário, secundário e terciário são atendidos pacientes que correm risco de morte, destacando-se como causas mais comuns as doenças cardiovasculares, emergências pediátricas e agravos por causas externas.

Os eventos cardiovasculares são considerados a principal causa de morte na população brasileira, enquanto as causas externas, incluindo causas intencionais e não intencionais, constam como a principal causa de morte nas primeiras quatro décadas de vida no Ceará e no Brasil, sendo inseridos neste contexto os acidentes de trânsito ${ }^{3}$.

No Estado do Ceará, para uma população de mais de 8 milhões de habitantes, não existe cobertura completa do Sistema de Atendimento Médico de Urgência (Samu) em todo o território estadual, sendo quehá apenas três hospitais terciários de referência para traumas mais complexos. Além disso se verifica desorganização nas redes de referência, acarretando sobrecarga nosprontos-socorros dos hospitais terciários. Finalmente, observa-se ainda falta de qualificação técnica dos profissionais de saúde para a prestação de serviços neste setor $^{4,5}$.
Em resumo, na maioria dos hospitais de pequeno porte do interior do Estado, onde é executado o primeiro atendimento, não há material básico adequado para os primeiros-socorros ${ }^{6}$. Faltam ainda recursos humanos qualificados ${ }^{4}$ e transporte apropriado para o encaminhamento dos casos mais graves às instituições de referência dentro da rede do sistema de saúde do Estado, obedecendo aos princípios da regionalização ${ }^{7}$.

Nesse contexto, seria impossível organizar o currículo de um novo curso médico no Estado do Ceará que não fosse capaz de proporcionar, na formação do egresso, elementos capazes de transformar esta realidade. Esses elementos não poderiam se restringir à maior inserção curricular do conteúdo emergências médicas, mas acima de tudo seria preciso envolver os estudantes, capacitá-los eficientemente, desenvolver autonomia e habilidades de tomada de decisão. Ou seja, formar um novo médico demandaria uma revisão da tendência histórica de formação dos profissionais de saúde, pautada no uso de metodologias conservadoras (ou tradicionais), sob forte influência do mecanicismo de inspiração cartesiano-newtoniana, fragmentado e reducionista ${ }^{8,9}$. Nessa prática, o aluno é pouco motivado para resolver problemas, buscar informações, refletir sobre a sua prática e desenvolver autonomia. Outro ponto crítico é que a maioria das escolas possui um modelo educacional pouco direcionado para as necessidades de saúde prioritárias da população, geralmente concentrado no enfrentamento das doenças, desenvolvido de forma pouco integrada, centrado somente nos aspectos biológicos. Os alunos, muitas vezes, têm dificuldades de entender o impacto relativo dos fatores ambientais, sociais e comportamentais na etiologia e progressão das enfermidades, porque frequentemente não estão familiarizados com o contexto de vida dos pacientes e com os serviços de saúde. Considerando-se que a graduação dura somente alguns anos, enquanto a atividade profissional pode permanecer por décadas, e que os conhecimentos e competências vão se transformando velozmente, torna-se essencial utilizar uma metodologia de ensino adequada, que resultena formação de profissionais de saúde capazes de compreender o aprender a conhecer, o aprender a fazer, o aprender a conviver e o aprender a ser, garantindo a integralidade da atenção à saúde com qualidade, eficiência e resolutividade ${ }^{9,10}$.

As metodologias ativas estão alicerçadas em um princípio teórico significativo, a autonomia, algo explícito na invocação de Paulo Freire ${ }^{11}$, numa perspectiva de transformação da realidade; afinal, conhecer é transformar ${ }^{11,12}$. Este tipo de metodologia de ensino-aprendizagem tem permitido a articulação entre a universidade, o serviço e a comunidade, por possibilitar uma leitura e intervenção consistente sobre a realidade; valorizar todos os atores no processo de construção coletiva e 
seus diferentes conhecimentos; promover a liberdade no processo de pensar e no trabalho em equipe, superando a dicotomia que há entre a formação e a prática profissional, buscando modelos em que a formação acadêmica incorpore as práticas do sistema de saúde, bem como as características e especificidades das comunidades nas quais provavelmente os futuros profissionais irão se inseriri, ${ }^{9,13,14}$.

Um segundo ponto a ser destacado como desafio e limitação para o ensino de emergências na graduação é a utilização dos pacientes para vivenciar e aprender os procedimentos e condutas, além da obrigação de fornecer o tratamento ideal e garantir a segurança e bem-estar dos doentes. Equilibrar essas duas necessidades representa uma tensão ética fundamental na educação médica, sobretudo no cenário da emergência, onde a gravidade dos doentes e o risco de morte são uma constante. A aprendizagem baseada em simulação pode ajudar a atenuar esta tensão por meio do desenvolvimento de habilidades e atitudes dos profissionais de saúde, ao mesmo tempo em que há proteção dos pacientes contra riscos desnecessários.

A aprendizagem significativa baseada em simulação foi institucionalizada em outras profissões de alto risco, como aviação, energia nuclear, e entre os militares, maximizando a segurança e minimizando o risco. Durante muito tempo, a aplicação de simulação no ensino médico foi subutilizada por uma série de razões, incluindo custo, falta de prova rigorosa de efeito eresistência à mudança. Recentemente, o movimento internacional de segurança do paciente proporcionou uma atmosfera mais receptiva à expansão do uso de simuladores na formação médica, em face da publicação de grandes estudos epidemiológicos que descreveram lesões evitáveis e inaceitáveis para os pacientes como resultado do tratamento médico. Existem evidências da utilização de simulação desde os primórdios da medicina. No século XVI, manequins (referidos comophamtons) foram desenvolvidos para ensinar técnicas de obstetrícia e reduzir as altas taxas de mortalidade materna e infantil. Hoje, é comum para os estudantes fazer suas primeiras injeções em uma laranja, sutura em pedaços de esponjase ensaiar entrevistas médicas ou exame físico entre colegas. Estudos recentes têm apoiado a eficácia dos simuladores de baixa e alta fidelidade no reforço de habilidades relacionadas a procedimentos e a atitudes comportamentais e sociais em medicina $^{15}$

Em 2006, foi iniciado na Universidade de Fortaleza (Unifor) o curso de Medicina, seguindo as Diretrizes Curriculares Nacionais, tendo a aprendizagem baseada em problemas como metodologia de ensino e destacando as necessidades de saúde do Estado do Ceará como elemento norteador na composição dos conteúdos curriculares. Especificamente no que diz respeito ao ensino da temática emergência, optou-se pela inserção do tema em formato longitudinal, iniciando-se no terceiro semestre com as ações de suporte básico à vida, avançando gradualmente pelo quarto semestre e culminando com o módulo Habilidades Médicas em Emergências distribuído no sétimo e oitavo semestres ${ }^{16}$. A confecção destes últimos módulos de emergências iniciou-se em 2009 após a realização de uma pesquisa epidemiológica no final de 2008 sobre a situação do atendimento médico de emergência em uma microrregional do interior do Estado do Ceará, ${ }^{4,5,17}$. Esta região agrupa oito municípios de pequeno porte populacional, com atuação expressiva do Programa Saúde da Família, sete hospitais de pequeno porte eum hospital secundário,cujo hospital terciário se situa a mais de $100 \mathrm{~km}$ dos referidos municípios. A amostragem retrata a realidade das outras 21 microrregiões do Estado.

Com base nesta análise de situação, foram identificadas as principais carências e problemas de saúde relacionados ao atendimento de emergência. Os achados subsidiaram a construção dos objetivos de aprendizagem a serem abordados no sétimo (S7) e oitavo (S8) semestres do curso de Medicina da Unifor.

A carga horária proposta foi de 2 horas semanais em Laboratório de Habilidades Médicas(LH), complementadas por atividades curriculares em ambientes de prática clínica: plantões no Serviço de Atendimento Médico de Urgências (Samu-Fortaleza) para o S7, e para o S8 atendimento supervisionado por professores da Universidade em Emergências Clínica e Cirúrgica de hospitais terciários da capital e numa emergência pediátrica de um hospital secundário, também na capital.

A metodologia de ensino utilizada no cenário do LH inclui a discussão de casos a partir da simulação de situações de atendimento de emergência. $\mathrm{O}$ processo de avaliação é contínuo e diversificado, de caráter formativo e somativo, utilizando instrumentos padronizados, além da ênfase sobre a reflexão acerca da própria prática (através da técnica de debriefing $\left.{ }^{18,19}\right)$ modificada para a nossa realidade.

Oriundo de um relato de experiência, este artigo visa descrever o planejamento e a implantação dos módulos de emergência médica de um curso de Medicina do Nordeste do Brasil, utilizando daavaliação dos discentes como ferramenta de gestão para aprimoramento dos referidos módulos.

\section{MÉTODOS}

Com o objetivo de inserir o aluno no contexto real das emergências médicas, os módulos foram desenvolvidos em três etapas: planejamento, implantação (ensino-aprendizagem-avaliação) e avaliação do módulo pelos discentes. 
$\mathrm{Na}$ fase de planejamento, ocorreram reuniões entre os professores responsáveis pelo sétimo e oitavo semestres. A sistematização dos conteúdos ea abordagem metodológica foram discutidas a partir dos achados da análise de situação realizada, seguindo a matriz curricular do curso de Medicina daUnifor.

A metodologia de ensino e aprendizagem proposta pelo projeto pedagógico do curso é o Aprendizado Baseado em Problemas, que integra o conteúdo entre as várias estratégias educacionais do curso: Grupo Tutorial (GT), Laboratório Morfofuncional (LMF) e Assistência Integrada à Saúde (AIS). No Laboratório de Habilidades (LH), especificamente no S7, o estudante vivencia, utilizando simuladores de alta fidelidade e pacientes simulados, casos-problema encenadosque abordam as principais causas de emergência no contexto do Estado do Ceará, dentre elas insuficiência cardíaca e infarto do miocárdio, relacionadas ao uso de crack e cocaína, o grande queimado, maus-tratos na infância e adolescência, afogamento, atendimento pré-hospitalar ao politraumatizado, avaliação, abordagem e conduta no politraumatizado com obstrução de via aérea, traumatismo cranioencefálico, trauma raquimedular, trauma torácico, abdominal e pélvico, trauma musculoesquelético e vascular, trauma pediátrico, trauma na gestante e em Geriatria.

No semestre seguinte (S8), também integrado com as estratégias educacionais do GT, LMF e AIS, o conteúdo trabalhado envolve as emergências clínicas mais comuns, iniciando-se com as situações de emergência em Pediatria: intoxicação exógena, cetoacidose diabética, convulsão febril, meningite, diarreia e desidratação, pneumonia e aspiração/engasgo por corpo estranho e reanimação cardiorrespiratória do neonato. Seguem-se as emergências da gestante, especificamente as emergências hipertensivas (pré-eclâmpsia, eclâmpsia e pico hipertensivo). E, finalmente, as emergências clínicas no adulto: síndrome coronariana aguda, taquicardias, bradicardias, atendimento a parada cardiorrespiratória (fibrilação ventricular/taquicardia ventricular sem pulso, assistolia e atividade elétrica sem pulso) e acidente vascular cerebral.

O plano de ensino é fornecido aos alunos no início de cada semestre. Nele constam os objetivos de aprendizagem de todo o módulo, assim como a lista de todos os assuntos a serem abordados no semestre, sendo um tema diferente a cada semana. Cada tema é problematizado na forma de caso clínico, com as questões de aprendizagem estabelecidas e respondidas pelo aluno previamente à abordagem do caso. Segue-se o atendimento pelos alunos, no papel de médico, do caso simulado em manequins ou pacientes atores, dentro das estruturas do LH na respectiva semana. Cada turma prática possui 20 alunos que são supervisionados por dois ou três professores.

Nas aulas práticas do S7, o ambiente simula um pronto-socorro com todos os materiais necessários ao atendimento de emergência. A montagem do cenário clínico específico varia de acordo com o caso da semana. São utilizados manequins, simuladores ou paciente simulado previamente instruído. A dinâmica da prática desenvolve-se inicialmente com a realização do atendimento por um dos alunos, enquanto os outros observam e anotam os pontos mais relevantes do atendimento, estabelecendo-se uma avaliação crítica, pontuando as falhas e acertos na conduta do colega. A professora responsável pelo caso apenas acompanha e atua quando é necessário, respondendo algum questionamento sobre o exame físico do paciente simulado ou manequim utilizado. Após o atendimento, são comentadas todas as etapas, passo a passo, sobre a sistematização e conduta adotada pelo aluno. Inicialmente, o aluno que atuou se autoavalia, em seguida os colegas que o assistiram, e por último a professora. Neste momento, a professora refaz todo o atendimento, enfatizando as etapas mais importantes, conforme previsto pelos objetivos de aprendizagem determinados no plano de ensino. Alguns pontos que, ao final, não ficaram bem sedimentados ficam como tarefa acadêmica para serem trazidos no encontro seguinte, a exemplo da farmacologia dos agentes indicados na prescrição do paciente.

A vivência em ambiente de prática clínica real no S7 é realizada mediante plantão noturno no Samu - Sistema de Atendimento Móvel de Urgência, onde os alunos em estágio curricular acompanham as equipes médicas nas Unidades de Suporte Avançado (UTI móvel) e na Central de Regulação.

A estratégia de aprendizagem do S8 possui dinâmicas de práticas em LH semelhantes às organizadas no $\mathrm{S7}$, com algumas diferenças no que diz respeito ao atendimento nas semanas de reanimação cardiorrespiratória. Nestas semanas, os alunos formam grupos de quatro e atendem a uma situação clínica de parada cardiorrespiratória em um espaço onde há gravação e transmissão em tempo real para uma outra sala onde estão os 16 alunos restantes da turma. Ao final do atendimento, um dos alunos comunicará a má notícia de um desfecho desfavorável do atendimento a um ator previamente instruído, que estará atuando como acompanhante do paciente atendido. Esta comunicação é transmitida também ao vivo para o restante da turma. Ao final, o vídeo é revisto por todos os estudantes. Os professores conduzem a discussão sobre a sistematização do atendimento pelo grupo, comentando o desempenho dos alunos individualmente e no grupo, como também o ator descreve a sua percepção acerca da comunicação da má notícia. Ainda no S8, os alunos realizam práticas 
supervisionadas na Emergência do Hospital Geral de Fortaleza, tanto nos setores de Clínica Médica como de Cirurgia, além de estágio no maior hospital de trauma da capital e na emergência pediátrica de um hospital secundário. Em cada uma dessas emergências hospitalares cinco alunos permanecem durante um período de quatro semanas supervisionados por um professor.

\section{Os processos de avaliação no S7 e S8}

A avaliação desempenha papel crucial no processo de ensino e aprendizagem, sobretudo quando existe a necessidade de avaliar domínios além do cognitivo. Deve apresentar critérios de validade e confiabilidade que assegurem a aquisição das competências estipuladas para o estudante naquele período de estudo. Tal complexidade requer muito mais do que um único tipo ou técnica de avaliação pode proporcionar. Ao mesmo tempo, não se pode perder de vista a função de aprendizagem que a avaliação encerra. Desta forma, apesar de todos os instrumentos comporem uma nota final somativa, os momentos de feedback ${ }^{20}$ são regulares e repetidos no decorrer do semestre.

Assim, o processo avaliativo inclui múltiplos instrumentos de aferição. São realizados dois testes cognitivos com questões de múltipla escolha e discursivas, abordando o conteúdo teórico e prático com níveis taxonômicos variados ${ }^{21}$, sendo o aluno convidado predominantemente a analisar, julgar situações e tomar decisões. Os testes ocorrem em dois momentos, em meados e no final do semestre. Nesse segundo momento, é realizado um Osce (exame clínico objetivo estruturado) ${ }^{22} \mathrm{com}$ oito a dez estações.

Um instrumento padronizado de avaliação longitudinal, preenchido para cada aluno pelos professores do LH, contabiliza as presenças e as tarefas acadêmicas como parte integrante do processo de avaliação, incluindo-se neste momento um forte olhar sobre a dimensão atitudinal.

Nos cenários de prática clínica, são também realizadas avaliações. No Samu, o aluno é avaliado a cada plantão nos critérios pontualidade, interesse em aprender e relação entre os membros da equipe de atendimento, relação aluno-paciente e disponibilidade para auxiliar quando solicitado. Nas emergências dos hospitais terciário e secundário, a avaliação é aplicada no final de cada rodízio de quatro semanas. $\mathrm{O}$ instrumento de avaliação padronizado trabalha critérios importantes no desenvolvimento do profissionalismo na prática, tais como: assiduidade, pontualidade, comportamento e atitude na relação com o paciente e seus familiares, trabalho em equipe, motivação, organização. O desempenho individual do estudante é fornecido pelos professores no formato de feedback ${ }^{20}$ imediatamente após as avaliações.
O processo se encerra com a avaliação do módulo, no final de cada semestre, realizada pelos alunos por meio de questionários específicos. Os dados coletados nesta avaliação subsidiam as reuniões de revisão do módulo que ocorre sempre entre os semestres, a fim deimplementar melhorias e garantir a excelência da aprendizagem significativa em emergências. Todos os estudantes são convidados a responder ao questionário com questões abertas e fechadas, mas nem todos o fazem. Nos anos de 2009, 2010 e 2011, os referidos módulos foram avaliados pelos discentes, sendo cada turma composta por 60 alunos.

Os itens objetivos de cada questionário de acordo com sua área temática foram analisados como variáveis individuais. As respostas às questões abertas foram analisadas quanto à frequência e à importância dada na justificativa dos sujeitos e foram apresentadas seguindo a técnica de análise de conteúdo ${ }^{23,24}$, que se diz ser "[...] um conjunto de técnicas de análise das comunicações que utiliza procedimentos sistemáticos e objetivos de descrição do conteúdo das mensagens"'(p. 49).

A tabulação dos dados foi efetuada nos programasEpiInfo versão 3.5.1 e Microsoft Office Excel 2003. Posteriormente, foi efetivado o tratamento e análise dos respectivos dados mediante a adoção dos softwares Microsoft Office Excel 2003 eSPSS Statistics 17.0.

\section{RESULTADOS E DISCUSSÃO}

A primeira turma de Medicina da Unifor concluiu o curso em julho de 2012.As informações acerca das atitudes e compromisso dos alunos nos ambientes por onde estagiaram durante o internato são motivadoras para o corpo docente. No primeiro semestre de 2012, 17 alunos que estavam no internato foram aprovados em processo seletivo para Unidades de Pronto Atendimento (UPA) da Secretaria de Saúde do Estado do Ceará.

A avaliação dos alunos em relação ao módulo é um importante instrumento de validação entre o que foi planejado e pretendido pelos professores na montagem do plano de ensino e o que foi realmente percebido pelos alunos no desenvolver das atividades do semestre, principalmente em se tratando de um curso que estava em processo de implantação.Foi usado um questionário com perguntas fechadas, utilizando-se como resposta a escala de Likert(insuficiente, requer grandes melhorias $=2$; fraco, requer melhorias $=4$; regular $=6$; bom $=$ 8 ; excelente $=10$ ) e três perguntas abertas sobre pontos fortes do módulo, pontos que demandam melhorias e sugestões de melhorias.

Em 2009, 31 alunos da primeira turma do S7 avaliaram o módulo, atribuindo as respectivas médias de notas nos se- 
guintes quesitos: conteúdo teórico-prático $=7,48$; importância do módulo $=7,86$; qualidade do módulo $=6,96$; organização geral $=5,92$; carga horária no Laboratório de Habilidades (2 horas $)=4,12$. No campo destinado ao papel do professor,as médias de notas foram as seguintes nos referidos quesitos: domínio do assunto $=7,66$; clareza e objetividade na exposição $=7,22$; capacidade de prender a atenção $=7,02$; esclarecimento de dúvidas e estímulo à participação dos discentes $=7,08$. Os principais aspectos positivos referidos foram: (i) conteúdo do módulo, que, segundo os discentes, é "importante para a formação", "bom", "relevante"e "objetivo"; (ii) qualidade dos professores:"motivou o estudo dos alunos", "foi incrementada pela presença de especialistas no assunto", "os professores eram habilitados"; (iii) métodos de ensino: "apresentavam inovação didática", "eram bons", "estimulavam o estudo prévio" e "apresentavam dinâmicas produtivas, que fomentavam a discussão em grupo"; (iv) práticas: "simulavam a realidade" e "foram essenciais, contribuindo para a formação". Já os principais pontos de melhoriareferidos foram: (i) carga horária reduzida, que "não permitia praticar muito", "insuficiente para que todo o conteúdo fosse visto e também praticado"; (ii) organização dos horários ruim; (iii) avaliações referentes às discussões dos casos, "que eram excessivas"; (iv) material para aprendizagem prática, que, segundo alguns discentes, "era insuficiente para algumas práticas". Como sugestão principal para melhoria do módulo, alguns discentes indicaram mais práticas em ambientes hospitalares de emergência ou no Samu.

Após avaliação das sugestões pelo coordenador do módulo e pelo grupo gestor do curso,foram implementadasmudanças a fim de melhorar o S7 e não repetir os erros na construção do S8. As ações foram relacionadas predominantemente à aquisição de maior quantidade de material e disponibilização de mais horários para aprendizagem no laboratório, assim como maior integração entre os cenários de prática e as atividades do LH,ea inclusão de uma professora especialista em Cardiologia, com atuação em emergências cardiológicas.

Algumas dessas mudanças surtiram efeito na construção do S8, e,no final de 2010, a mesma turma de alunos anteriormente no S7, agora no S8, se posicionoude forma mais positiva diante do módulo de emergência do S8. Trinta e três alunos participaram da avaliação,e a média das notas foi a seguinte: conteúdo teórico-prático $=7,72$; qualidade geral $=$ 7,63; importância do módulo para a formação médica $=9,42$; carga horária no LH (2 horas/semana) = 6,12; organização geral $=6,57$. Acerca da avaliação prática (Osce), realizadano final do semestre letivo, foram obtidas as seguintes médias: metodologia $=8,27$; qualidade geral $=8,18$; organização ge- ral $=8,06$. Os principais aspectos positivos referidos foram: (i) conteúdo, considerado pelos discentes como "importante para a formação", "bom", "importante por ter emergências cardiológicas na grade" e "de grande aplicabilidade prática"; (ii) casos clínicos:"voltados para a prática ", "baseados na realidade" e "de objetivos claros"; (iii) métodos de ensino: "inovadores, dinâmicos", "bons", "interessantes por estimularem a discussão"; (iv) práticas: "eram estimulantes", "simulavam a realidade". Já os principais pontos de melhoria mencionados foram: (i) carga horária reduzida, que "não permitia que todos praticassem", "não era bem dividida quanto à discussão e prática". Como sugestão principal para melhoria, alguns discentes referiram que a carga horária deveria ser aumentada.

Em 2011, foi realizada uma nova avaliação geral do módulo do sétimo semestre, dessa vez por 57 dos 60 alunos. Obtiveram-se os seguintes resultados: quanto aabrangência, quantidade de tópicos, nível de profundidade: 64,9\% dos alunos caracterizaram como excelente; relevância dos temas: $70,2 \%$ excelente; alcance dos objetivos de aprendizagem previstos:66,7\% excelente; nível de integração entre as atividades do próprio módulo: 70,2\% excelente; integração entre as atividades dos outros módulos (GT, LMF, AIS): 47,4\% bom, 35,1\% excelente.Os principais aspectos positivos referidos foram: (i) conteúdo do módulo:"relevância dos temas, aprendizagem prática em cada aula"; (ii) qualidade dos professores: "professoras atenciosas, didáticas", "interesse das professoras", empenho das professoras"; (iii) métodos de ensino: "práticas realizadas interessantes e reais"; (iv) práticas: "as melhores do curso até o momento".

Entende-se que o processo ensino-aprendizagem é complexo, apresenta caráter dinâmico e não acontece de forma linear, como um somatório de conteúdos acrescidos aos anteriormente estabelecidos ${ }^{9,25}$. Exige ações direcionadas para que o discente aprofunde e amplie os significados elaborados mediante sua participação, enquanto requer do docente o exercício permanente do trabalho reflexivo, da disponibilidade para o acompanhamento, da pesquisa e do cuidado, que pressupõe a ocorrência de situações imprevistas e desconhecidas ${ }^{9}$. O ato de ensinar-aprender deve ser um conjunto de atividades articuladas, nas quais esses diferentes atores compartilham, cada vez mais, parcelas de responsabilidade e comprometimento.

Na educação libertadora e autônoma, o estudante precisa assumir um papel cada vez mais ativo, se descondicionando da atitude de mero receptor de conteúdos, buscando efetivamente conhecimentos relevantes para os problemas e objetivos da aprendizagem. Iniciativa criadora, curiosidade científica, espírito crítico reflexivo, capacidade para autoavaliação, cooperação para o trabalho em equipe, senso de responsabi- 
lidade, ética e sensibilidade na assistência são características fundamentais a serem desenvolvidas em seu perfil ${ }^{9}$. Retirar o discente do seu cômodo papel de espectador não constitui tarefa simples, principalmente em se tratando hoje da geração multimídia, que frequenta os bancos escolares. No entanto, o docente precisa encarar este desafio como o único formato de ensino que realmente promove aprendizagem. Tornar o estudante participante dos processos de revisão dos módulos é uma forma efetiva de, pelos menos parcialmente, responsabilizar o aluno pela construção do seu saber.

A utilização da simulação incorpora realidade à aprendizagem de forma segura e controlada, e foi observada pelos alunos como elemento diferencial nos módulos. A simulação é uma forma de ensino e desenvolvimento da aprendizagem que vem se difundindo nas escolas médicas do mundo há mais de 40 anos. As pesquisas históricas e contemporâneas apontam as 12 ações que contribuem para as boas práticas de educação médica baseada em simulação: (1) feedback;(2) prática deliberada; (3) integração curricular; (4) avaliação dos resultados; (5) simulação fidedigna; (6) aquisição de habilidades e manutenção; (7) domínio da aprendizagem; (8) transferência para a prática; (9) trabalho em equipe; (10) avaliações;(11) capacitação e desenvolvimento dos instrutores;(12) contexto educacional e profissional. A prática de simulação na educação é um serviço complexo de intervenção que precisa ser planejado e praticado com atenção e contexto organizacional ${ }^{26}$. Há evidências de que o ensino médico baseado em simulação é superior à educação médica tradicional na aquisição de habilidades específicas ${ }^{27}$. No Laboratório de Habilidades Médicas do curso de Medicina da Unifor, esta vem sendo a diretriz. Se os estudantes egressos a partir deste modelo de aprendizagem atuarão como elementos transformadores da atual realidade do atendimento das emergências médicas no Estado do Cearáainda é uma incógnita que o tempo vai responder.

\section{CONCLUSÃO}

Este trabalho descreveu o processo de planejamento, estruturação e implantação do ensino de emergências médicas contextualizado para a realidade atual do Estado do Ceará,com base em metodologias ativas, abordagem prática em ambiente de simulação e vivência em estágios supervisionados no Samu e Pronto Atendimento Hospitalar. Avaliou-se o processo de ensino-aprendizagem na perspectiva dos discentes, que, no decorrer dos semestres, foi considerado de forma progressiva como satisfatório,com base nas mudanças realizadas. A inovação em si não consistesomente na utilização de metodologias ativas e de simulação, masna flexibilidade curricular que parte da observação das necessidades do sistema de saúde e da percepção dos discentes e docentes, estruturando o objeto por meio da construção dialogada de todas as partes envolvidas: comunidade, sistema de saúde, docentes e discentes.

\section{REFERÊNCIAS}

1. Brasil. Ministério da Saúde. Política Nacional de Atenção às Urgências.3.ed. ampl. Brasília: Editora do Ministério da Saúde; 2006. p.256.

2. Morin E. Introdução ao pensamento complexo. 2.ed. Lisboa: Instituto Piaget; 1990.

3. Reichenheim ME, Souza ER, Moraes CL, Mello Jorge MHP, Silva CMFP, Minayo MCS. Violência e lesões no Brasil: efeitos, avanços alcançados e desafios futuros. The Lancet. [online].2011 [capturado 20 set. 2011]; 75-89. Disponível em: http:/ /download.thelancet.com/flatcontentassets / pdfs/brazil/brazilpor5.pdf

4. Fernandes CR, Mendes WA, Lourinho PRV, Silva ICR, Muniz LMMM. Percepção dos diretores de hospitais municipais de uma microrregional do Ceará sobre recursos humanos e materiais disponíveis para o primeiro atendimento a vítimas de trauma. Anais do XXV Outubro Médico; 2010 out. 13-16; Fortaleza, Brasil. Revista Ceará Médico. Fortaleza: Associação Médica Cearense; 2010. p.52.

5. Messias KLM, Fernandes CR, Mendes WA, Santos JPL, Iannini NG, Sappi LFF. Perfil e percepção dos médicos que prestam atendimento hospitalar a vítimas de trauma na microrregional 13 do estado do Ceará. Anais do XXV Outubro Médico; 2010 out. 13-16; Fortaleza, Brasil. Revista Ceará Médico. Fortaleza: Associação Médica Cearense; 2010. p.52.

6. Freitas ER, Fernandes CR, Sousa EM, Silva LES, Albuquerque Filho DA, Guimaraes DF. Análise dos atendimentos hospitalares por causas externas na microrregional 13 do estado do Ceará. Anais do XXV Outubro Médico; 2010 out. 13-16 ;Fortaleza, Brasil. Revista Ceará Médico. Fortaleza: Associação Médica Cearense; 2010. p.38.

7. Ceará. Secretaria de Saúde. Políticas de Saúde. Organização de Serviços. Regionalização; 2010. [Acessado em 20 set 2011]. Disponíveis: http:/ / www.saude.ce.gov.br/site/ index.php?option=com_content\&view $=$ article\&id=761\&I temid $=203$

8. Capra F. O ponto da mutação: a ciência, a sociedade e a cultura emergente. Cultrix: São Paulo; 2006

9. Mitre SM, Batista RS, Mendonça JMG. Metodologias ativas de ensino-aprendizagem na formação profissional em saúde: debates atuais. Ciênc. saúde Colet 2008;13(2):21332144 . 
10. Fernandes JD, Ferreira SLA, Oliva R, Santos S. Diretrizes estratégicas para a implantação de uma nova proposta pedagógica na Escola de Enfermagem da Universidade da Federal da Bahia. Rev. Enfermagem 2006;56(54):392-395.

11. Freire P. Educação e mudança. São Paulo: Paz e Terra; 1999.

12. Freire P. Pedagogia da autonomia: saberes necessários à prática educativa. 33.ed. São Paulo: Paz e Terra; 2006.

13. Feuerwerker LCM. Gestão dos processos de mudança na graduação em medicina. In: Marins JJN, Rego S, Lampert J, Araújo JGC. Educação Médica em Transformação: instrumentos para a construção de novas realidades. São Paulo: Hucitec; 2004. 390p.

14. Feurwerker LCM, Sena RR. A contribuição ao movimento de mudança na formação profissional em saúde: uma avaliação das experiências UNI. Interface - Comunic., Saúde, Educ 2006;6(10):37-50

15. Amitai Z, Wolpe PR, Stephen D, Glick S. Simulation-Based Medical Education: An Ethical Imperative, Academic Medicine 2003;78(8):783-788.

16. Fernandes CR, Arnaud FCS, Gomes JMA, Correia RV, Escalante RD, Bessa OC. Inserção longitudinal do conteúdo de emergência no currículo do curso de medicina da UNIFOR. Anais do $48^{\circ}$ Congresso Brasileiro de Educação Médica, 2010, out 27-30;Goiânia, Brasil. Revista Brasileira de Educação Médica. Rio de Janeiro: Associação Brasileira de Educação Médica, ABEM, 2010;34:142-142.

17. Fernandes CR, Messias KLM, Lopes NAP, Portela NMB. Mortalidade por causas externas na macrorregião de Sobral-Ceará: um grave problema de saúde pública. Anais do IX Congresso Brasileiro de Saúde Coletiva, 2009, out 31 - Nov 04. Recife. Ciênc. saúde colet. Rio de Janeiro : Hucitec-Abrasco, 2009;suplem.

18. Raemer D, Anderson M, Cheng A, Fanning R, Nadkarni V, Savoldelli G. Research regarding debriefing as part of the learning process. SimulHealthc. 2011;6 Suppl:S52-7.

19. Dieckmann P, MolinFriis S, Lippert A, OstergaardD. The art and science of debriefing in simulation: Ideal and practice. MedTeach. 2009;31(7):287-94.

20. Zeferino AMB, Domingues RCL, Amaral E. Feedback como estratégia de Aprendizado no Ensino Médico. Rev Bras Educ Med 2007;31(2):176-179

21. Ferraz APCM, Belhot RV. Taxonomia de Bloom: revisão teórica e apresentação das adequações do instrumento para definição de objetivos instrucionais. Gestão e Produção, São Carlos 2010;17(2):421-431.

22. Carraccio C, Englander R. The objective structured clinical examination: a step in the direction of competency-based evaluation. Arch Pediatr Adolesc Med.2000;154(7):736-41.
23. Oliveira DC. Análise de conteúdo temático-categorial: uma proposta de sistematização. Rev. Enferm. UERJ 2008;16(4):569-576

24. Silva C, Gobbi B, Simão A. O uso da análise de conteúdo como uma ferramenta para a pesquisa qualitativa: descrição e aplicação do método. Organ. Rurais Agroind, Lavras, 2005;7(1):70-81.

25. Cyrino EG, Toralles-Pereira ML. Trabalhando com estratégias de ensino-aprendizado por descoberta na área da saúde: a problematização e a aprendizagem baseada em problemas. Cad Saúde Pública 2004;20(3):780-788.

26. Mcgaghie WC, Issenberg SB, Petrusa ER, Scalese RJ. A critical review of simulation-based medical education research: 2003-2009. Med Educ 2010; 44:50-63.

27. Mcgaghie WC, Issenberg SB, Cohen ER, Barsuk JH, Wayne DB. Does simulation-based medical education with deliberate practice yield better results than traditional clinical education? A meta-analytic comparative review of the evidence. Acad Med 2011;86:706-711.

\section{CONTRIBUIÇÃO DOS AUTORES}

Cláudia Regina Fernandes - Idealizou, implantou e desenvolveu os módulos de Emergências Médicas da Universidade de Fortaleza, participou da coleta de dados, análise dos dados, avaliação, escrita do artigo e revisão; Sandra Nívea dos Reis Saraiva Falcão - Participou do desenvolvimento dos módulos, análise dos dados, avaliação e escrita do artigo; Josenília Maria Alves Gomes - Participou da escrita do artigo e revisão; Fernando Barros Colares - Participou da coleta dos dados, análise dos dados, escrita do artigo; Maria Márcia Morais Souto Maior - Participou do desenvolvimento dos módulos, avaliação e escrita do artigo; Rafaela Vieira Correa - Participou da escrita do artigo e revisão; Olívia Andre Alencar Costa Bessa - Participou da escrita do artigo e revisão.

\section{CONFLITO DE INTERESSES}

Declarou não haver.

\section{ENDEREÇO PARA CORRESPONDÊNCIA}

Cláudia Regina Fernandes

Rua Marcelino Lopes, 4520 - casa 9

Sapiranga - Fortaleza

CEP 60833-075 - CE

E-mail: clauregifer@gmail.com 